\title{
In vitro evaluation of anti-infective activity of a Lactobacillus plantarum strain against Salmonella enterica serovar Enteritidis
}

\author{
Jugal Kishore Das ${ }^{1}$, Debasmita Mishra ${ }^{1}$, Pratikshya Ray ${ }^{1}$, Prangya Tripathy ${ }^{1}$, Tushar K Beuria ${ }^{2}$, Neera Singh ${ }^{1}$
} and Mrutyunjay Suar ${ }^{1 *}$

\begin{abstract}
Background: Salmonella enterica serovar Enteritidis infections are known to exhibit worldwide prevalence with increased morbidity and mortality. The conventional strategies like antibiotic therapy and vaccination have not only proved to be of sub-optimal efficacy but also led to the development of multidrug resistant strains of Salmonella. Antimicrobial activities of probiotics against various enteropathogens and other health promoting effects have assumed greater significance in recent years. The present study aims to evaluate the efficacy of a Lactobacillus plantarum strain (KSBT 56, isolated from a traditional food product of India), in preventing Salmonella enterica serovar Enteritidis growth and pathogenicity in vitro.

Methods and results: The cell free culture supernatant (CFCS) of KSBT 56 strain notably inhibited the growth of Salmonella Enteritidis without affecting the growth of other gram-positive lactic acid bacteria. The isolated KSBT 56 strain produces lactic acid similar to other standard probiotic strains like Lactobacillus plantarum MTCC 1407. The free radical production by KSBT 56 strain was studied by using sodC mutant of $S$. Enteritidis, which exhibited reduced growth in the presence of CFCS of the KSBT 56 strain, indicating the inhibitory activity of free radicals on the growth of $S$. Enteritidis. Our results also showed a significant reduction in the biofilm forming ability of Salmonella Enteritidis in the presence of the KSBT 56 strain (2 log cfu/ml, p = 0.01). Further, the anti-infective characteristics of KSBT 56 strain was validated by gentamicin protection assay which revealed $80 \%$ reduction in the invasion of Salmonella Enteritidis to HCT-116 cell line (Salmonella Enteritidis and KSBT 56 in a 1:1 ratio) and delayed addition of Salmonella Enteritidis by $1 \mathrm{~h}$. Similarly, the reduced adhesion of Salmonella to the HCT-116 cells was observed along with the down regulation of hilA gene of Salmonella Pathogenicity Island 1 (SPI1) indicating that they might have acted synergistically to decrease the invasion of the pathogen into the cell line.

Conclusions: KSBT 56 strain effectively inhibited the growth, invasion and the biofilm forming ability of Salmonella Enteritidis without inhibiting the growth of other Lactobacillus strains. Overall, our result suggested that KSBT 56 can be used as a potential probiotic strain with considerable beneficial effects on the host.
\end{abstract}

Keywords: Probiotic, Lactobacillus, Salmonella, HCT-116, Cell free culture supernatant

\footnotetext{
* Correspondence: msbiotek@yahoo.com

'School of Biotechnology, KIIT University, Bhubaneswar 751024, India

Full list of author information is available at the end of the article
} 


\section{Background}

Salmonella enterica is a major food borne pathogen and one of the leading causes of serious illness ranging from acute gastroenteritis to systemic infections like typhoid. Infections with non typhoidal serovars of Salmonella enterica, predominantly Salmonella Enteritidis (S. Enteritidis) and $S$. Typhimurium are more frequent and occur in both developing and industrialized nations. These infections are primarily associated with gastrointestinal inflammation and diarrhea and are generally self-limiting [1]. The established strategies to combat Salmonella infections include vaccination and the use of antibiotics. However, the frequent and prolonged use of antibiotics not only leads to increasing antibiotic resistance among Salmonella serovars but also alters the intestinal commensal flora [2]. The emergence of multidrug resistant strains and the suboptimal efficacy of currently available vaccines have necessitated the search for alternative therapies against Salmonella infections [3-5]. One such promising alternative is the possible therapeutic use of probiotics against various enteropathogens [6-8].

Probiotics are defined as "live microorganisms which when administered in adequate amounts confer a health benefit on the host" [3]. The possible mechanisms by which probiotics may inhibit enteric pathogens include modification of the host intestinal environment and immune system, competition for nutritional substrates as well as sites of adhesion on intestinal epithelial cells, secretion of antimicrobial compounds and inactivation of toxins [4]. Earlier studies have reported the use of probiotics in the prevention and treatment of gastrointestinal infections caused by Salmonella [5]. However, the underlying molecular mechanisms by which probiotics offer protection against gastrointestinal pathogens are not fully elucidated [9-11]. The most extensively studied probiotic strains are reported from genera Lactobacillus and Bifidobacterium, which are also included in many functional foods and dietary supplements $[12,13]$. The beneficial effects of the probiotics are known to be genus, species and strain specific and a particular probiotic strain is found to be active against selected enteric pathogen only $[9,14,15]$. The selection of a microbial strain is therefore, an important criterion to consider it as a probiotic for its effective and potential therapeutic use.

Food based probiotics have assumed greater significance in recent years as different food products can harbor native and beneficial Lactobacilli and thus can be used for both nutritional and therapeutic purposes. Traditional Indian foods are well known for their unique fermentation style and can be used as a source of potentially beneficial probiotics. The antibacterial mechanisms of action of these Lactobacillus strains, especially the production of nonbacteriocin molecules, have not been extensively studied. The main objective of the present study is to determine the efficacy of an isolated probiotic strain in preventing $S$. Enteritidis infections. Further, the mechanism of antimicrobial activity was assessed in order to establish it as a potential probiotic strain, specifically active against $S$. Enteritidis, which contributes to major Salmonella infections.

\section{Results}

\section{Effect of CFCS on viability of S. Enteritidis}

The CFCS of Lactobacillus strains are known to have antimicrobial effects against enteric pathogens [14]. In this study, the effect of CFCS of KSBT 56 strain on $S$. Enteritidis viability was assessed using flow cytometric analysis. The dead bacterial cells were shown in the propidium iodide quadrant (Figure 1). Different concentrations $(3 \%, 5 \%, 7 \%, 9 \%$ and $11 \%)$ of CFCS of KSBT 56 were used to study its effect on the viability of $S$. Enteritidis. The inhibition of $S$. Enteritidis increased with increasing concentration of the CFCS of the probiotic strain. Effective killing of $S$. Enteritidis (89.6\%) was observed with $11 \%$ of CFCS after $4 \mathrm{~h}$. Similarly, CFCS was also found to be effective against other pathogens such as E. coli, S. Typhi and $S$. Typhimurium (data not shown).

\section{Effect of CFCS of the KSBT 56 on other Lactobacillus strains}

Probiotics should be able to selectively inhibit pathogens while not having any deleterious effect on the normal gut flora, to be considered as safe for consumption. The effect of CFCS of the KSBT 56 strain was studied by incubating different Lactobacillus strains with the probiotic CFCS. No significant differences were observed in the viability of the standard Lactobacillus strains in the presence or absence of the CFCS (Figure 2). The results partly confirm the safety of KSBT 56 as a probiotic strain as it does not have a deleterious effect on the normal commensal gut flora.

\section{Production of lactic acid by KSBT 56}

Lactic acid is one of the important factors produced by Lactobacillus strains which inhibit various pathogens at a specific concentration. For example, the standard Lactobacillus strain Lactobacillus plantarum (L. plantarum) MTCC 1407 inhibits pathogens like Salmonella and Shigella, at a concentration of $6.0 \mathrm{mM}$ lactic acid produced at $6 \mathrm{~h}$. In the present study, the lactic acid concentration of KSBT 56 was estimated to be $5 \mathrm{mM}$ at $6 \mathrm{~h}$, which was comparable to that of $L$. plantarum MTCC 1407. The total lactic acid concentration in the CFCS of KSBT 56 was also estimated to be $5 \mathrm{mM}$ at $6 \mathrm{~h}$, indicating its antimicrobial property. 

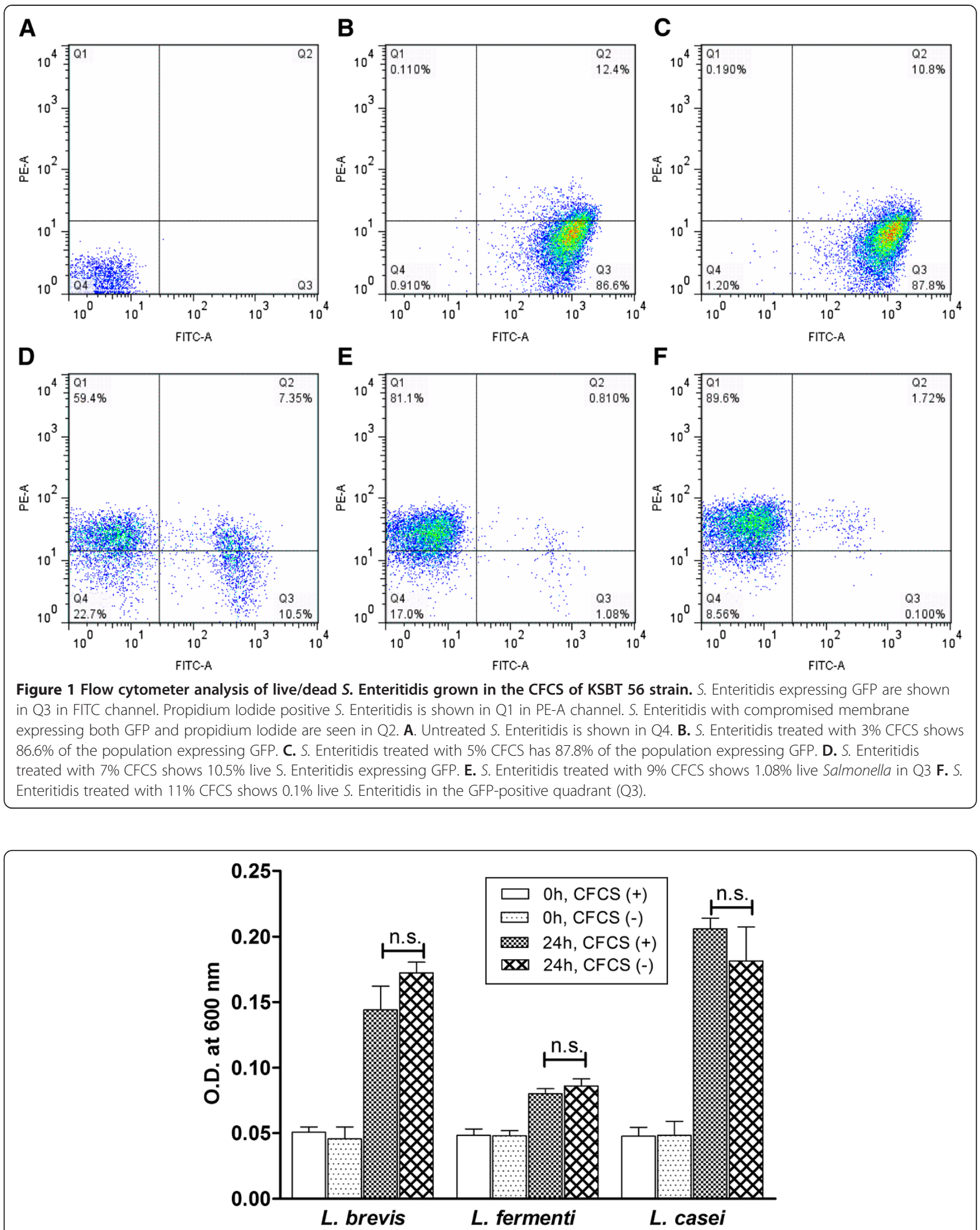

Figure 2 The growth of different Lactobacillus strain in the presence of CFCS of KSBT 56 strain. The absorbance of the cultures at $600 \mathrm{~nm}$ is plotted on the $\mathrm{y}$-axis. The growth of different Lactobacillus strains was analysed by comparing absorbance at $0 \mathrm{~h}$ and $24 \mathrm{~h}$ of growth in presence of CFCS of KSBT 56. 
Inhibitory effect of free radicals produced by KSBT 56 on S. Enteritidis

The antimicrobial activity of the free radicals produced by KSBT 56 strain against $S$. Enteritidis was determined by using superoxide dismutase $(\operatorname{sod} C)$ gene knock out mutant. A sodC mutant is known for its increased susceptibility to free radicals as compared to the wild type (WT) strain. Results showed that $S$. Enteritidis harboring sodC mutation exhibited reduced growth in the presence of CFCS of the KSBT 56 strain in the co-culture experiment (Figure 3). As compared to the $S$. Enteritidis (WT) strain, $\triangle$ sodC mutant was sensitive to CFCS treatment showing a 2-log decrease in its viability on the addition of CFCS $(\mathrm{p}=0.01)$. This indicates that $S$. Enteritidis is susceptible to the free radicals produced by the KSBT 56 strain.

\section{Inhibitory effect of KSBT 56 on biofilm formation ability of S. Enteritidis}

The effect of KSBT 56 on the biofilm forming ability of $S$. Enteritidis was determined by co-culture experiment and by delayed addition of Salmonella to KSBT 56 strain in a 96 well plate. Biofilm formation was confirmed by crystal violet staining (data not shown). The cfu recovered from the biofilm formed by Salmonella in a 96 well plate were plated on LB agar plates in different dilutions. The simultaneous addition of $S$. Enteritidis with the KSBT 56 strain did not show any significant inhibition of the biofilm formation by $S$. Enteritidis. However, on the delayed addition $(1 \mathrm{~h})$ of $S$. Enteritidis to the culture containing the probiotic strain, a 2-log decrease in biofilm forming colonies of Salmonella was observed ( $\mathrm{p}=0.01$ ) (Figure 4).

\section{Inhibition of invasion of S. Enteritidis by KSBT 56}

To determine the inhibitory effect of KSBT 56 on invasion of $S$. Enteritidis, standard gentamicin protection assay was

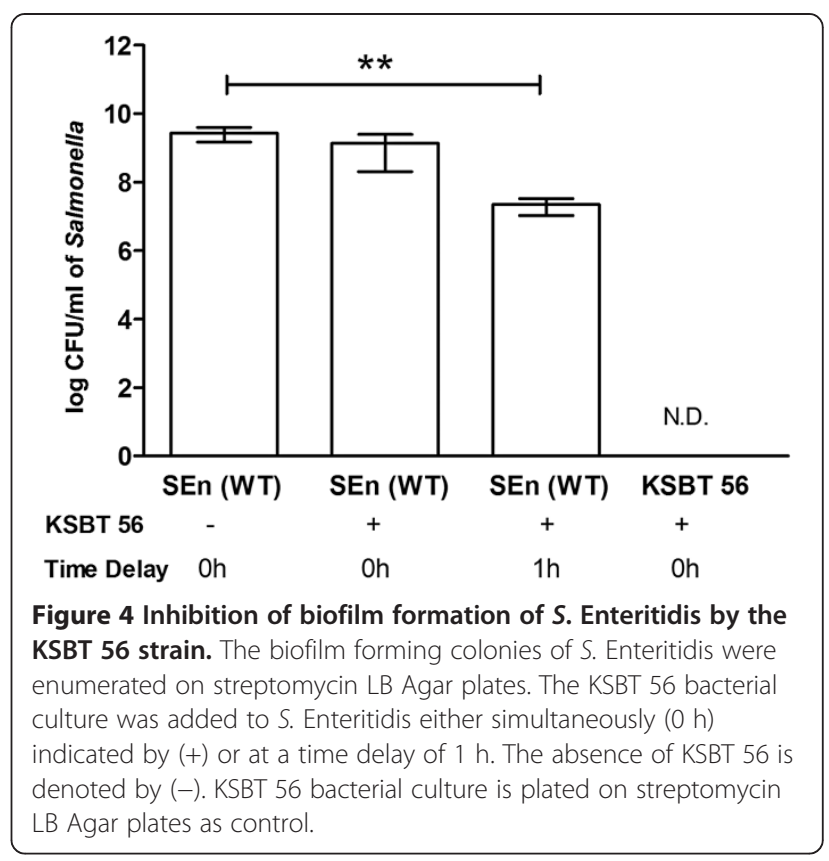

performed with simultaneous and delayed addition of $S$. Enteritidis strain to HCT-116 cell line. Gentamicin kills the extracellular bacteria while the intracellular bacteria are plated on LB agar plates and cfu enumerated. Reduced invasion (by $40 \%$ ) of $S$. Enteritidis was observed on simultaneous addition of the pathogen and the probiotic strain in the ratio of 1:1 (Figure 5A). Further, the invasion efficiency of $S$. Enteritidis was significantly reduced by $80 \%$ on addition of KSBT 56 strain $1 \mathrm{~h}$ prior to the addition of $S$. Enteritidis as compared to the control ( $S$. Enteritidis only) ( $\mathrm{p}=0.0012)$. Similarly, the invasion of Salmonella was reduced by $23 \%$ on co-incubation with CFCS of KSBT 56 strain and by $28 \%$ on delayed addition of $S$.
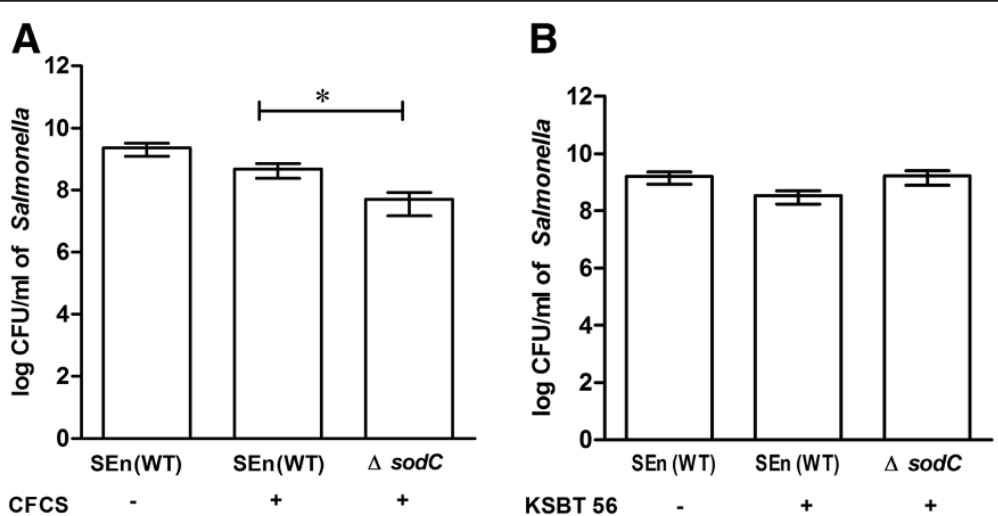

Figure 3 Inhibition of growth of S. Enteritidis WT and $\Delta$ sodC mutant in the presence of CFCS (A) of KSBT 56 or live KSBT 56 (B). A. S. Enteritidis (SEn) WT or a mutant strain deficient of sodC gene $(\triangle$ sodC) were co-incubated with CFCS. B. The above groups were also co-incubated with live KSBT 56 bacterial culture. The cfu was enumerated by plating on LB agar plates supplemented with streptomycin. The presence of CFCS or KSBT 56 is indicated by $(+)$ and the absence is indicated by $(-)$. The growth of $\Delta$ sodC is compared with S. Enteritidis WT strain grown in the presence of CFCS or live KSBT 56 strain. 

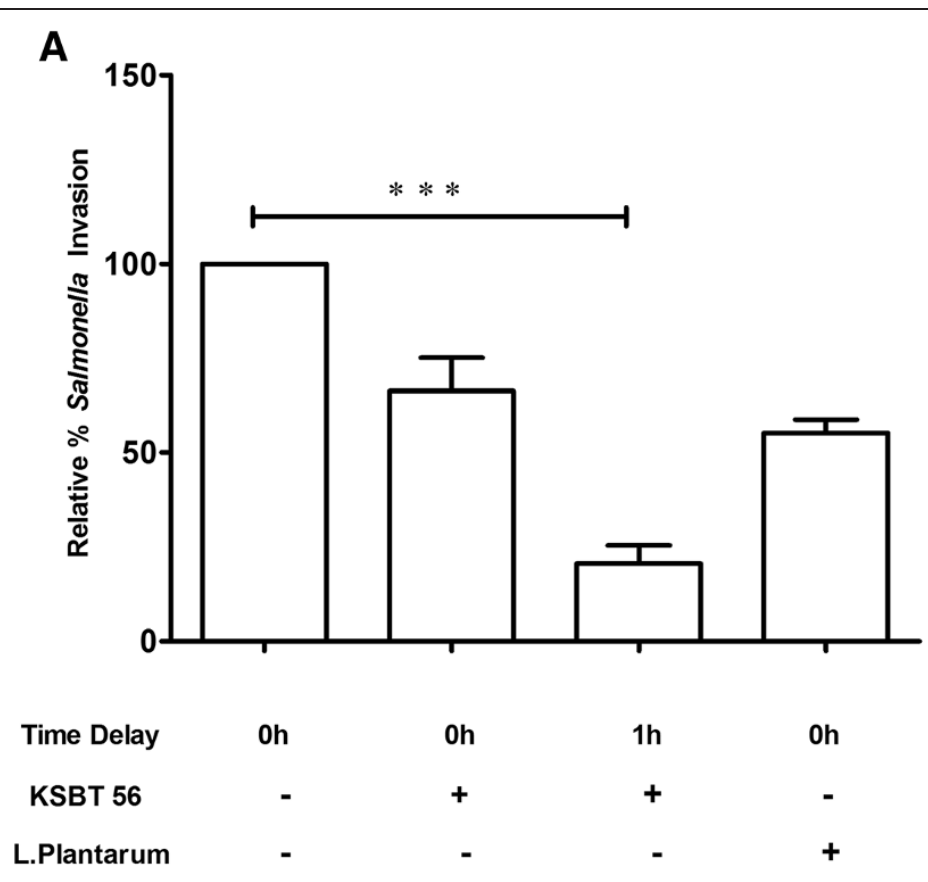

\section{B}

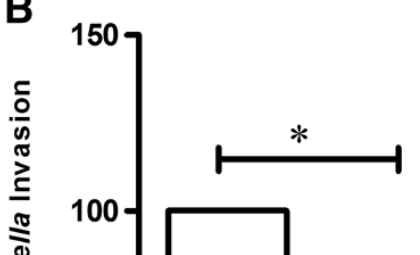

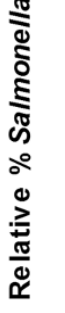

\section{Time Delay}

CFCS- KSBT 56

CFCS- L.Plantarum

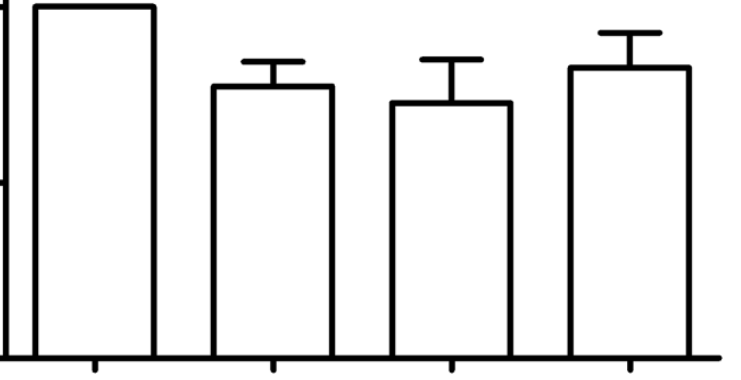

$\mathrm{Oh}$

Oh
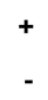

1h
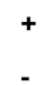

Oh

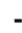

$+$

Figure 5 Effect of KSBT 56 on invasion of S. Enteritidis (A) and effect of CFCS of KSBT 56 on invasion of S. Enteritidis to HCT-116 cells. A. Gentamicin protection assay was performed to determine the invasion of S. Enteritidis into the HCT-116 cell line in the presence (+) or absence (-) of KSBT 56 strain. The pathogen and the KSBT 56 strain were either co-infected together into the cell line $(0 \mathrm{~h})$ or the pathogen was added at a time delay of $(1 \mathrm{~h})$. B. The effect of CFCS on invasion of S. Enteritidis was determined by co-incubating $\mathrm{S}$. Enteritidis with the CFCS of KSBT 56 in 24- well tissue culture plate seeded with HCT-116 cell line. S. Enteritidis was also cultured with CFCS for $1 \mathrm{~h}$ before infection of HCT-116 cells. L. plantarum MTCC 1407 was taken as a reference strain. The invasion of S. Enteritidis to HCT-116 cells is taken as control.

Enteritidis after incubation of the pathogen with CFCS for $1 \mathrm{~h}$ (Figure 5B). The confocal images provide further conclusive evidence of the reduced invasion of $S$. Enteritidis and adherence of the KSBT 56 strain to the HCT-116 cell line (Figure 6A-D).
Adhesion of S. Enteritidis to HCT-116 cell line in the presence or absence of KSBT 56

Probiotics are known to adhere to intestinal epithelial cells thereby competitively excluding the adhesion of pathogens. The adhesion of $S$. Enteritidis to HCT-116 colon 

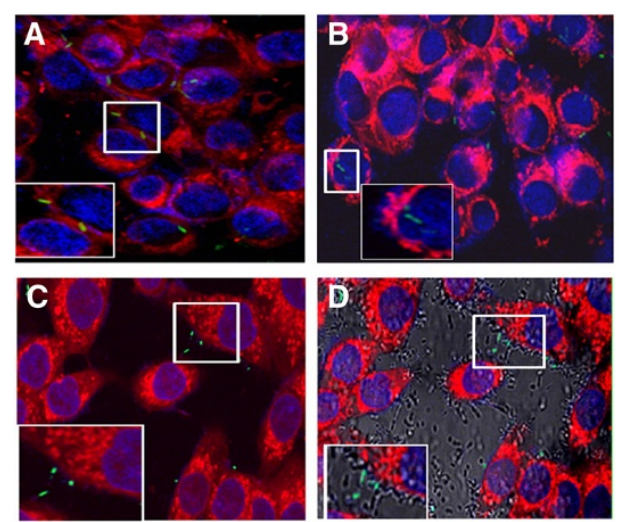

Figure 6 Confocal images of Salmonella invasion (A-D) and Expression of hilA gene by RT-PCR (E). Confocal images were taken at $63 \mathrm{X}$ magnification using Leica CLSM. The membrane of HCT-116 cell lines were stained with plasma red dye and S. Enteritidis was tagged with GFP. The KSBT 56 strain was observed in phase contrast. A. S. Enteritidis invasion into HCT-116 in the absence of KSBT 56 strain. B. S. Enteritidis coinfected with KSBT 56 strain into HCT-116 cell line shows reduced invasion of S. Enteritidis. C. Delayed addition of S. Enteritidis after addition of KSBT 56 strain by $1 \mathrm{~h}$ further reduces the invasion of Salmonella into the cell lines. D. Merged image of panel C with phase contrast shows KSBT 56 adhering to HCT-116. E. RT-PCR analysis of hilA gene of S. Enteritidis grown in increasing concentration of CFCS of KSBT 56 strain. L. plantarum MTCC 1407 is a reference strain. The fold change in the expression of hilA gene is compared to S. Enteritidis WT (Untreated). SEn refers to S. Enteritidis and LP refers to L. plantarum MTCC 1407.

epithelial cell line was studied by simultaneous and delayed addition of $S$. Enteritidis and KSBT 56 strain. No significant reduction in the adhesion of the $S$. Enteritidis to HCT-116 cells was observed on simultaneous addition of the probiotic and the pathogenic strain. However, the adhesion of $S$. Enteritidis to HCT-116 cell line was significantly reduced $(\mathrm{p}=0.01)$ on the delayed infection of Salmonella by $1 \mathrm{~h}$ after the addition of the KSBT 56 strain. The CFCS of KSBT 56 strain did not decrease the adhesion of $S$. Enteritidis to HCT-116 cell line significantly either on co-incubation or on delayed addition of the pathogen, after $1 \mathrm{~h}$ of incubation with CFCS of KSBT 56 strain. The percentage of adhesion of KSBT 56 and $S$. Enteritidis is shown in Table 1.

\section{Effect of CFCS on hilA (SPI1) expression}

SPI1 encodes genes which are involved in the invasion of Salmonella into intestinal epithelial cells. The hilA gene, a major transcriptional regulator of SPI1, is reportedly down regulated in the presence of probiotic CFCS
[16]. RT-PCR was used to study hilA gene expression in the presence of varying concentrations of CFCS of KSBT 56 strain. The results showed that with the increasing concentration of CFCS of the KSBT 56 strain, hilA gene expression was consistently down regulated. The hilA gene expression in the presence of CFCS of KSBT 56 strain is shown in Figure 6E. Thus, apart from reduced adhesion, the down regulation of hilA gene was also responsible for the reduced invasion of $S$. Enteritidis to HCT-116 cells as shown in Figure 6A-D.

\section{Discussion}

Probiotics have been successfully used for the prevention and treatment of various gastrointestinal diseases of human and animals [17]. The beneficial Lactobacillus strains present in the fermented dairy products are known to have a nutritional and therapeutic effect on human health [18]. Several in vivo and in vitro studies have demonstrated that probiotics can inhibit Shigella dysenteriae [19], Salmonella [5] and Clostridium difficile

Table 1 Adhesion of S. Enteritidis and KSBT 56 to HCT-116 cell line

\begin{tabular}{|c|c|c|}
\hline Infection of HCT-116 cell line & Adhesion percentage of SEn & Adhesion percentage of KSBT 56 \\
\hline S. Enteritidis WT : KSBT 56 (1:1) & $11.68 \% \pm 3.68$ & $6.43 \% \pm 1.69$ \\
\hline S. Enteritidis WT : KSBT 56 (1:1) delayed addition of S. Enteritidis by $1 \mathrm{~h}$ & $7.7 \% \pm 1.09^{\mathrm{a}}$ & $7.53 \% \pm 2.4$ \\
\hline S. Enteritidis WT & $12.08 \% \pm 1.51^{b}$ & N.T \\
\hline KSBT 56 & N.T & $8.0 \% \pm 2.34$ \\
\hline S. Enteritidis + CFCS of KSBT 56 & $10.35 \% \pm 3.50$ & N.T \\
\hline S. Enteritidis incubated with CFCS of KSBT 56 for $1 \mathrm{~h}$ & $10.06 \pm 1.06$ & N. T \\
\hline
\end{tabular}

The values represent the S.D of 3 different experiments. NT is not tested. SEn is Salmonella Enteritidis.

The significant differences between $\mathrm{a}$ and $\mathrm{b}$ were calculated using $t$-test. 
[20] associated diarrhea. However, the basis of their mode of action has largely remained unanswered. The present study, therefore aimed to understand the underlying mechanism of action of a novel Lactobacillus strain isolated from a fermented milk product.

In this study, the CFCS of the isolated KSBT 56 strain, inhibited $S$. Enteritidis growth in in vitro culture system and the live KSBT 56 culture effectively prevented its attachment and invasion to the colon epithelial cell lines (HCT-116). Flow-cytometric dead/live staining analysis is a sensitive measure of bacterial cell death. Therefore the technique has been used in our study to assess $S$. Enteritidis viability when cultured in the presence of CFCS of KSBT 56 strain. By increasing the concentration of CFCS, viable counts of $S$. Enteritidis decreased consistently, but at low concentration, a significant percentage of $S$. Enteritidis were also observed showing positive for both GFP and propidium iodide. This is probably because of their compromised status of the membrane integrity. Similarly, no deleterious effects were observed on the other commensal gut flora further establishing its safety profile. Previous studies have reported that CFCS of L. plantarum induces complete inhibition of Salmonella growth, which was mainly attributed to the lactic acid production by the probiotic strain [11,21]. CFCS of probiotic Lactobacillus strains reportedly contain several antimicrobial compounds [22], lactic and non-lactic acids as well as hydrogen peroxide which can kill various enteropathogens [23]. Earlier studies have also established that lactic acid production by probiotic Lactobacillus strains is a key mechanism involved in inhibiting bacterial growth [24,25]. In the present study, the lactic acid produced by the KSBT 56 strain was comparable to that of the reference strain $L$. plantarum and therefore we suggest that the inhibitory activity shown against $S$. Enteritidis could be partly due to the production of lactic acid in the CFCS. Alternatively, the mechanism of the antimicrobial activity of probiotic Lactobacillus strains might also include production of other non-lactic acid components and peroxide radicals $[17,25]$. Furthermore, to study the effect of free radical produced by the strain KSBT 56, sodC mutation was incorporated in $S$. Enteritidis. Results showed the increased inhibition of growth of $\triangle \operatorname{sod} C$ mutant when cultured in the presence of CFCS, indicating that free radicals might be generated by the KSBT 56 strain.

One of the reasons Lactobacilli have been widely studied is because of their remarkable ability to inhibit the growth of various pathogens by producing antimicrobial compounds and inhibiting biofilm formation by various pathogens [26]. The inhibitory effect of Lactobacillus supernatant on biofilm formation by $K$. pneumonia was shown in a recent study [27]. Similarly, in another related study, the inhibitory effect of $L$. fermentum supernatant was observed on the Klebsiella growth and biofilm formation [28]. In the present study, similar results were observed, where the biofilm forming ability of the pathogen was reduced on delayed addition of Salmonella, in the presence of KSBT 56 strain.

One of the key steps identified in the pathogenesis of intestinal pathogens is their ability to attach to the surfaces of intestinal epithelial cells via fimbriae or pili, present on the bacterial cell surface [29]. The subsequent step in Salmonella pathogenesis after attachment is the invasion of intestinal epithelial cells. In the present work, colon epithelial cell line, HCT-116 was used to study the adherence and invasion of $S$. Enteritidis. Our results demonstrated a significant reduction in the adherence of Salmonella to the HCT-116 cell lines when incubated with the KSBT 56 strain. Similarly, invasion of $S$. Enteritidis to HCT-116 cell line was noticeably reduced both in the co-culture experiment and on the delayed addition of Salmonella. The significant reduction in invasion of $S$. Enteritidis on delayed addition of the pathogen may be due to the initial attachment of KSBT 56 strain to colon epithelial cells further preventing the attachment of Salmonella. Previous studies have also reported reduced adhesion of pathogens in the presence of probiotic strains due to competitive exclusion of the pathogens [30]. However, in the present study we observed reduced invasion of Salmonella to HCT-116 cells by $40 \%$ in co-culture experiment, although there was no significant difference in the adhesion of KSBT 56. The CFCS of KSBT 56 strain also inhibited the invasion of Salmonella, while not having any significant effect on the adhesion of the pathogen to HCT-116 cell line. These results collectively indicate the involvement of an alternative mechanism besides the competitive exclusion of the pathogen, thereby reducing the invasion of $S$. Enteritidis into colon epithelial cell lines.

The invasion of intestinal epithelial cells by Salmonella requires a set of genes present on the SPI1, the expression of which is tightly regulated by hilA [31]. Previous studies have reported that various probiotic components down regulate hilA gene expression in $S$. Typhimurium thereby preventing its invasion into intestinal epithelial cells [16,31-33]. Our data was also in agreement with these findings and showed down regulation of hilA gene expression of $S$. Enteritidis in the presence of CFCS of KSBT 56. Based on these results, we proposed that the CFCS of the KSBT 56 strain might secrete components which can down regulate virulence related genes in $S$. Enteritidis. The reduced expression of genes involved in invasion is therefore one of the important mechanisms which contributes to the antimicrobial effect of probiotics on intestinal pathogens. Overall, the results obtained from this study indicate that, the KSBT 56 strain isolated from fermented milk product can serve as 
a putative probiotic with effective anti-microbial properties. The in vitro data suggests that the isolated KSBT 56 strain might exert its beneficial effect via multifactorial mechanisms, which might act synergistically to antagonize intestinal pathogens. In the present study, we have provided key insights into possible mechanism of action of the KSBT 56 strain against $S$. Enteritidis and established its beneficial properties as a probiotic strain, which can be further exploited for commercial purposes.

\section{Conclusions}

Overall results from this study suggested that KSBT 56 strain showed a potent antimicrobial activity against $S$. Enteritidis. The KSBT 56 strain was found to considerably inhibit the growth, adherence and invasion of $S$. Enteritidis. Similarly, the biofilm forming ability of $S$. Enteritidis was substantially reduced by the KSBT 56 strain. The structures of the nonbacteriocin and nonlactic acid components and the specificity of their antagonistic activity against enteroinvasive and enterovirulent $S$. Enteritidis strain remain an important area of future research.

\section{Methods}

Bacterial strains and culture conditions

KSBT 56 strain was isolated from dahi chenna (traditional fermented milk product) obtained from a local household. L. plantarum MTCC 1407 was used as a reference strain. Lactobacillus strains were grown in deMan, Rogosa and Sharpe (MRS) (HiMedia Pvt. Ltd., Mumbai) broth under aerobic conditions at $37^{\circ} \mathrm{C}$ for $18 \mathrm{~h}$. S. Enteritidis was grown for $12 \mathrm{~h}$ and subcultured in Luria-Bertani LB (HiMedia Pvt. Ltd., Mumbai) at $37^{\circ} \mathrm{C}$ and used until they reached the early log phase of growth. For, biofilm, adhesion and invasion assays, equivalent $\mathrm{cfu} / \mathrm{ml}$ counts of live KSBT 56 and $S$. Enteritidis cultures were used to determine the competitive exclusion of the pathogen and a sub-lethal dose of CFCS was used to determine the effect of CFCS on adhesion and invasion of the pathogen. Preliminary experiments confirmed M-17 medium to be an appropriate medium for co-culture experiments with $S$. Enteritidis and the live KSBT 56 strain. The bacterial strains used in this study are listed in Table 2.

\section{Preparation of cell free culture supernatant}

CFCS of the probiotic strains are generally preferred over live probiotic bacteria for in vitro inhibition assays because probiotics have longer lag phase and generation time than S. Enteritidis. Further, Salmonella growth would be favoured before the probiotic strain could express its antimicrobial activity. Therefore, the CFCS of KSBT 56 strain was taken for the inhibition assays against $S$. Enteritidis. The CFCS of KSBT 56 strain was prepared as described by Truusalu et al. [6]. Briefly, cells were grown overnight in MRS broth for $18 \mathrm{~h}$. KSBT 56 culture was centrifuged at $15000 \mathrm{rpm}$ for $20 \mathrm{~min}$ and CFCS was filter-sterilized using 0.22 - $\mu \mathrm{m}$-pore-size millipore filters (Millipore Co., Italy).

\section{Cell cultures}

HCT-116 colon cells were grown in Dulbecco's modified Eagle Medium (DMEM) (HiMedia Pvt. Ltd., Mumbai) supplemented with $10 \%$ inactivated fetal bovine serum (FBS), glutamine $(1.5 \mathrm{mM} / 500 \mathrm{ml})$ and penicillin $(0.2 \mathrm{U} / \mathrm{ml})$, streptomycin $(0.1 \mathrm{mg} / \mathrm{ml})$. Cells were cultured at $37^{\circ} \mathrm{C}$ in an atmosphere of $5 \% \mathrm{CO}_{2}$ and $95 \%$ air.

\section{Effect of CFCS on viability of Salmonella}

$S$. Enteritidis culture transformed with pCJLA plasmid expressing green fluorescent protein (GFP) was grown overnight and subcultured for $2 \mathrm{~h}$. CFCS of the KSBT 56 strain was added in increasing concentration to the $S$. Enteritidis culture in an early exponential phase and incubated further for $3 \mathrm{~h}$. The bacterial cells were pelleted by centrifugation (1500 rpm for $5 \mathrm{mins}$ ) washed and resuspended in phosphate-buffered saline (PBS) and stained with propidium iodide. Flow cytometric analysis of the dead and live $S$. Enteritidis was carried out to analyze the inhibitory activity of the CFCS of the KSBT 56 strain. Flow cytometric measurements were performed using a FACScanto ${ }^{\mathrm{Tm}}$ II cytometer (BectonDickinson, Erembodegem, Belgium). First, unstained $S$. Enteritidis WT strains were used to set the photo multiplier tube (PMT) voltage of flow cytometer and distinguish bacteria from debris. Subsequently, S. Enteritidis expressing GFP and those stained with propidium iodide were detected on separate channels after setting the

Table 2 Bacterial strains used in the study

\begin{tabular}{lll}
\hline Strain name & Culture collection number & Reference or source \\
\hline S. Enteritidis P125109 & ATCC 13076 & {$[36]$} \\
Lactobacillus plantarum & MTCC 1407 & A kind gift from Dr. Knut Heller \\
KSBT 56 & NCDC 681 & Isolated from dahi chenna, a traditional food product of India \\
Lactobacillus fermenti & ATCC 9338 & ATCC \\
Lactobacillus brevis & ATCC 367 & ATCC \\
Lactobacillus casei & ATCC 9595 & A kind gift from Dr. Peter Leuthy \\
\hline
\end{tabular}


compensation control. The results were analyzed using Flowjo software (Vx 10.0.6 beta).

\section{Effect of CFCS of the isolated KSBT 56 strain on other Lactobacillus strains}

To determine the effect of the CFCS on other probiotic strains, overnight culture of Lactobacillus casei, Lactobacillus fermenti and Lactobacillus brevis were co-cultured with the CFCS of the probiotic strain at $37^{\circ} \mathrm{C}$ at minimum inhibitory concentration (11\% CFCS of KSBT 56) determined for $S$. Enteritidis earlier. The analysis of growth was based on OD measurements at $600 \mathrm{~nm}$ determined at baseline and after $24 \mathrm{~h}$ of incubation. Each experiment was performed in triplicates and repeated thrice.

\section{Determination of lactic acid concentration}

Lactic acid is the known component secreted by probiotic strains involved in the inhibition of enterocolitic pathogens. To determine whether the isolated KSBT 56 strain was producing lactic acid equivalent to other reference strains like L. plantarum MTCC 1407, a commercially available D- and L- Lactic acid estimation kit (Megazyme, Ireland) was used. After culturing the KSBT 56 and the reference strain for $6 \mathrm{~h}$ at $37^{\circ} \mathrm{C}$, lactic acid concentration was determined by D- and L- Lactic acid estimation kit according to manufacturers instructions. The lactic acid concentration in the CFCS of KSBT 56 strain was also estimated in a similar manner, to determine if the inhibitory activity of CFCS was due to the production of lactic acid.

\section{Determination of the antimicrobial activity of free radicals of the KSBT 56 strain}

To determine the antimicrobial activity of the free radicals produced by KSBT 56 strain against $S$. Enteritidis, superoxide dismutase gene $(\operatorname{sod} C)$ knock out mutant was used. sodC gene product is known to neutralize the effect of free radicals and protect the bacteria. One step inactivation method was used to construct a knock out mutant of $S$. Enteritidis WT by deleting the sodC gene [34]. Briefly, PCR primers providing homology to sodC gene were used to knock out the gene. An easily curable, low copy number plasmid pKD46 was used to facilitate homologous recombination of the PCR primers with homology to the sodC gene and template plasmid
(pKD4) carrying kanamycin resistance genes was transformed into $S$. Enteritidis. The primers used in the study are listed in Table 3. The mid log phase growth of $S$. Enteritidis WT strain and sodC gene knockout mutant was subcultured with 7\% CFCS of the KSBT 56 strain, for $4 \mathrm{~h}$. It was determined in earlier experiment that $7 \%$ CFCS of KSBT 56 strain considerably inhibited the growth of $S$. Enteritidis. Similarly, both the strains were co-cultured with the live KSBT 56 strain in M-17 medium. The cfu counts were enumerated by plating appropriate dilutions of the above groups in LB agar plates supplemented with streptomycin $(50 \mu \mathrm{g} / \mathrm{ml})$.

\section{Effect of KSBT 56 strain on biofilm formation}

The biofilm formation by $S$. Enteritidis was assessed by incubating Salmonella with the probiotic strain in a 96 well plate for $24 \mathrm{~h}$. The experiment was performed in the following groups: Group A: $S$. Enteritidis $\left(10^{8}\right.$ cells $\left./ \mathrm{ml}\right)$ Group B: $S$. Enteritidis + KSBT 56 strain in the ratio of 1:1. Group C: $S$. Enteritidis was added $1 \mathrm{~h}$ after the addition of the KSBT 56 strain in the ratio of 1:1. The biofilm formation by $S$. Enteritidis in the above wells was confirmed by crystal violet staining. The wells were washed with PBS thrice. Subsequently, the biofilm forming ability of Salmonella in various groups was determined by plating and enumeration of adherent bacteria in 96 well plates on LB Agar supplemented with streptomycin $(50 \mu \mathrm{g} / \mathrm{ml})$. The bacteria adhered to the wells forming biofilms were scrapped and different dilutions were plated. The plates were incubated at $37^{\circ} \mathrm{C}$ for $24 \mathrm{~h}$ and cfu count recovered from the biofilms was determined. KSBT 56 strain was included as a control in the experiment.

\section{Invasion assay}

Invasion of $S$. Enteritidis to HCT-116 cell line was carried out as previously described [35], with minor modifications. Briefly, HCT-116 cell line was maintained in DMEM and passaged until confluence. The monolayer cells were seeded on 24 well tissue culture plates (Nest Biotech, China) and the confluent cells were washed thrice with PBS. S. Enteritidis was grown overnight and subcultured for $4 \mathrm{~h}$ in LB medium [36]. Bacterial cells were washed and resuspended in DMEM and infected to HCT-116 cell lines at a multiplicity of infection (MOI)

Table 3 Primers used in the study

\begin{tabular}{|c|c|c|}
\hline Primer & Sequence $\left(5^{\prime}\right.$ to $\left.3^{\prime}\right)$ & Reference \\
\hline hilA F & TTAACATGTCGCCAAACAGC & [37] \\
\hline hilA R & GCAAACTCCCGACGATGTAT & [37] \\
\hline 16s rRNA F & GATCATGGCTCAGATTGAACGCTGGCGG & [37] \\
\hline $16 s$ rRNA R & CACCGCTACACCTGGAATTATACCCCCTC & [37] \\
\hline FwKOSenSodC & TITATGGGTAAAACGAAATTATGACGATATGGCTATGTTGCTGTGTGTAGGCTGGAGCTGCTTC & In this study \\
\hline RwKOSenSodC & TITATTAATGGTATTAACGATACAACCAAAAAACGAGGTAACTAATATGAATATCCTCCTTAGTT & In this study \\
\hline
\end{tabular}


of 100:1. The experiment was performed on 24 well plates in various groups. Group A: $S$. Enteritidis $\left(1 \times 10^{8}\right.$ cells $\left./ \mathrm{ml}\right)$ Group B: $S$. Enteritidis + KSBT 56 in the ratio of 1:1. Group C: $S$. Enteritidis was added $1 \mathrm{~h}$ after the addition of the KSBT 56 strain in the ratio of 1:1. Group D: $S$. Enteritidis + L. plantarum MTCC 1407 (1:1), was taken as control. The plate was incubated for $50 \mathrm{~min}$ at $37^{\circ} \mathrm{C}$ in $\mathrm{CO}_{2}$ incubator. HCT-116 cells were further incubated for $2 \mathrm{~h}$ in media containing gentamicin $(100 \mu \mathrm{g} / \mathrm{ml})$. Infected cells were washed twice with PBS and lysed with $0.1 \%$ Triton X-100. Dilutions of the resulting cell lysates were plated on streptomycin LB Agar for determination of intracellular bacterial counts. The above groups were also processed for confocal microscopy for supportive evidence of invasion assay. In a separate experiment, to determine the effect of CFCS on Salmonella invasion, S. Enteritidis was either co-incubated with CFCS (sub-lethal dose of 5\% of CFCS) or added after culturing with CFCS for $1 \mathrm{~h}$, to a 24-well tissue culture plate seeded with HCT-116 cells and standard gentamicin protection assay was performed as described above.

\section{Confocal microscopy}

HCT-116 monolayers were incubated overnight at $37^{\circ} \mathrm{C}$ in a humidified atmosphere at $5 \% \mathrm{CO}_{2}$ in cell culture medium without antibiotics before the addition of bacteria (MOI, 50:1). After incubation for $50 \mathrm{~min}$ in an appropriate medium without fetal bovine serum, cells were washed in PBS to remove non-invading bacteria. The monolayer cells, prepared on glass coverslips, in 24 well tissue culture plates (Nest Biotech, China), were fixed with $4 \%$ paraformaldehyde (PFA) and then stained with plasma red dye (Invitrogen, Green Island, USA). DAPI was used to stain the nucleus of HCT-116 cells. $S$. Enteritidis containing plasmid pCJLA expressing GFP was visualized using Confocal Laser Scanning Microscope (CLSM, Leica). Z-stacking was used to distinguish the internalized bacteria from the extracellular bacteria.

\section{Adhesion assay}

Adhesion assay was carried out as described previously [14]. Each well of a 24-well tissue culture plate was seeded with HCT-116 cells. $500 \mu \mathrm{l}$ of DMEM without serum and antibiotics was added to each well and incubated at $37^{\circ} \mathrm{C}$ for $30 \mathrm{~min}$. S. Enteritidis was grown overnight and the experiment was performed in the following groups. Group A: $S$. Enteritidis, $1 \times 10^{8} \mathrm{cfu} / \mathrm{ml}$ Group B: KSBT 56, $1 \times$ $10^{8} \mathrm{cfu} / \mathrm{ml}$ Group C: $S$. Enteritidis: KSBT 56 (1:1) Group D: $S$. Enteritidis added $1 \mathrm{~h}$ after the addition of KSBT 56. Plate was incubated for $20 \mathrm{~min}$ at $4^{\circ} \mathrm{C}$ and the cells were detached by adding Trypsin EDTA solution (HiMedia Pvt. Ltd., Mumbai). The cells were further incubated for $15 \mathrm{~min}$ at room temperature. The cell suspensions from each group were plated at appropriate dilutions on MRS agar and LB Agar supplemented with streptomycin for differential growth of KSBT 56 and S. Enteritidis. Similarly, the effect of CFCS on adhesion was determined by coincubating $S$. Enteritidis with CFCS in 24-well tissue culture plate seeded with HCT-116 cells or adding $S$. Enteritidis to the wells after $1 \mathrm{~h}$ of subculturing with CFCS, and adopting the above protocol of adhesion assay. A sub lethal dose of 5\% CFCS of KSBT 56 was used for the assay.

\section{Expression analysis of hilA gene (SPI1) by RT-PCR}

Probiotics are known to down-regulate the expression of virulence genes of $S$. Enteritidis present in both SPI1 and SPI2. hilA gene is the major transcriptional regulator of SPI1 and down-regulation of hilA reflects the downregulation of SPI1 genes required by $S$. Enteritidis for successful invasion into host epithelial cells [37]. To study the SPI1 regulation by KSBT 56, S. Enteritidis culture was grown overnight and subcultured for $4 \mathrm{~h}$ in the presence of increasing concentration of CFCS of the KSBT 56. RNA was isolated using Real Genomics RNA mini kit (Real Biotech Corporation, India) as per the manufacturers instructions and reverse transcribed to cDNA using cDNA synthesis kit (Fermentas, USA). The relative quantification of hilA gene expression was analyzed by using 16s rRNA as the reference gene for both treated and untreated $S$. Enteritidis culture. The RT-PCR was carried out by using SYBR Green Master Mix (Roche Applied Science, Mumbai, India). The PCR reaction conditions consisted of initial denaturation at $95^{\circ} \mathrm{C}$ for $5 \mathrm{~min}, 40$ cycles of denaturation at $95^{\circ} \mathrm{C}$ for $15 \mathrm{sec}$, followed by annealing at $54^{\circ} \mathrm{C}$ for $30 \mathrm{sec}$ and extension at $72^{\circ} \mathrm{C}$ for $45 \mathrm{sec}$. The primers used in the experiment are listed in Table 3.

\section{Statistical analysis}

All the data represent the mean \pm standard deviation of three independent experiments. The significant differences in the various experimental groups were determined by $t$ test with the help of GraphPad Prism software version 5. The flow cytometric data analysis was carried out by using Flowjo $\mathrm{V} \times 10.0 .6$ beta.

\footnotetext{
Abbreviations

S. Enteritidis: Salmonella enterica serovar Enteritidis; SEn: Salmonella Enteritidis PBS, Phosphate-Buffered Saline; SPI: Salmonella Pathogenicity Island; CFCS: Cell Free Culture Supernatant; CLSM: Confocal Laser Scanning Microscopy; L. plantarum: Lactobacillus plantarum; WT: Wildtype; GFP: Green Fluorescent Protein.

Competing interests

The authors declare that they have no competing interests.

Authors contributions

MS and JK conceived and designed the experiments. JK, DM and PR performed the experiments. TKB performed the confocal microscopy experiments. MS, NS and PT coordinated the study. NS and JK drafted the manuscript. All authors finally read and approved the manuscript.
} 


\section{Acknowledgement}

We thank Mr. Bhabani Sankar Sahoo of center for confocal microscopy at Institute of Life Sciences (ILS), Bhubaneswar for his excellent technical support in the microscopy unit. We acknowledge KIIT University for funding the project.

\section{Author details}

${ }^{1}$ School of Biotechnology, KIIT University, Bhubaneswar 751024, India.

${ }^{2}$ Institute of Life Sciences (ILS), Bhubaneswar 751024, India.

Received: 27 March 2013 Accepted: 1 May 2013

Published: 13 May 2013

\section{References}

1. Graham SM, Walsh AL, Molyneux EM, Phiri AJ, Molyneux ME: Clinical presentation of non-typhoidal Salmonella bacteraemia in Malawian children. Trans R Soc Trop Med Hyg 2000, 94(3):310-314.

2. Hammad AM, Shimamoto T: Towards a compatible probiotic-antibiotic combination therapy: assessment of antimicrobial resistance in the Japanese probiotics. J App/ Microbiol 2010, 109(4):1349-1360.

3. Bhan MK, Bahl R, Bhatnagar S: Typhoid and paratyphoid fever. Lancet 2005, 366(9487):749-762.

4. Birosova L, Mikulasova M: Development of triclosan and antibiotic resistance in Salmonella enterica serovar Typhimurium. J Med Microbiol 2009, 58(Pt 4):436-441.

5. Alcaine $S D$, Warnick LD, Wiedmann M: Antimicrobial resistance in nontyphoidal Salmonella. J Food Prot 2007, 70(3):780-790.

6. Truusalu K, Mikelsaar RH, Naaber P, Karki T, Kullisaar T, Zilmer M, Mikelsaar M: Eradication of Salmonella Typhimurium infection in a murine model of typhoid fever with the combination of probiotic Lactobacillus fermentum ME-3 and ofloxacin. BMC Microbiol 2008, 8:132.

7. Gibson GR, McCartney AL, Rastall RA: Prebiotics and resistance to gastrointestinal infections. Br J Nutr 2005, 93(Suppl 1):S31-34.

8. Roselli M, Finamore A, Britti MS, Mengheri E: Probiotic bacteria Bifidobacterium animalis MB5 and Lactobacillus rhamnosus GG protect intestinal Caco-2 cells from the inflammation-associated response induced by enterotoxigenic Escherichia coli K88. Br J Nutr 2006, 95(6):1177-1184

9. Reid G, Jass J, Sebulsky MT, McCormick JK: Potential uses of probiotics in clinical practice. Clin Microbiol Rev 2003, 16(4):658-672.

10. Collado MC, Isolauri E, Salminen S, Sanz Y: The impact of probiotic on gut health. Curr Drug Metab 2009, 10(1):68-78.

11. Rishi P, Mavi SK, Bharrhan S, Shukla G, Tewari R: Protective efficacy of probiotic alone or in conjunction with a prebiotic in Salmonella-induced liver damage. FEMS Microbiol Ecol 2009, 69(2):222-230.

12. Macpherson AJ, Harris NL: Interactions between commensal intestinal bacteria and the immune system. Nat Rev Immunol 2004, 4(6):478-485.

13. Frick JS, Schenk K, Quitadamo M, Kahl F, Koberle M, Bohn E, Aepfelbacher $M$, Autenrieth IB: Lactobacillus fermentum attenuates the proinflammatory effect of Yersinia enterocolitica on human epithelial cells. Inflamm Bowel Dis 2007, 13(1):83-90

14. Jacobsen CN, Rosenfeldt Nielsen V, Hayford AE, Moller PL, Michaelsen KF, Paerregaard A, Sandstrom B, Tvede M, Jakobsen M: Screening of probiotic activities of forty-seven strains of Lactobacillus spp. by in vitro techniques and evaluation of the colonization ability of five selected strains in humans. Appl Environ Microbiol 1999, 65(11):4949-4956.

15. Timmerman HM, Koning CJ, Mulder L, Rombouts FM, Beynen AC: Monostrain, multistrain and multispecies probiotics-A comparison of functionality and efficacy. Int J Food Microbiol 2004, 96(3):219-233.

16. Bayoumi MA, Griffiths MW: Probiotics down-regulate genes in Salmonella enterica serovar typhimurium pathogenicity islands 1 and 2. J Food Prot 2010, 73(3):452-460

17. Fayol-Messaoudi D, Berger CN, Coconnier-Polter MH, Lievin-Le Moal V, Servin AL: pH-, Lactic acid-, and non-lactic acid-dependent activities of probiotic Lactobacilli against Salmonella enterica Serovar Typhimurium. Appl Environ Microbiol 2005, 71(10):6008-6013.

18. Meyer AL, Elmadfa I, Herbacek I, Micksche M: Probiotic, as well as conventional yogurt, can enhance the stimulated production of proinflammatory cytokines. J Hum Nutr Diet 2007, 20(6):590-598.

19. Moorthy G, Murali MR, Devaraj SN: Protective role of lactobacilli in Shigella dysenteriae 1-induced diarrhea in rats. Nutrition 2007, 23(5):424-433.
20. Tejero-Sarinena S, Barlow J, Costabile A, Gibson GR, Rowland I: In vitro evaluation of the antimicrobial activity of a range of probiotics against pathogens: Evidence for the effects of organic acids. Anaerobe 2012, 18(5):530-538

21. Botes M, Loos B, van Reenen CA, Dicks LM: Adhesion of the probiotic strains Enterococcus mundtii ST4SA and Lactobacillus plantarum 423 to Caco- 2 cells under conditions simulating the intestinal tract, and in the presence of antibiotics and anti-inflammatory medicaments. Arch Microbiol 2008, 190(5):573-584.

22. Gopal PK, Prasad J, Smart J, Gill HS: In vitro adherence properties of Lactobacillus rhamnosus DR20 and Bifidobacterium lactis DR10 strains and their antagonistic activity against an enterotoxigenic Escherichia coli. Int J Food Microbiol 2001, 67(3):207-216.

23. Servin AL, Coconnier MH: Adhesion of probiotic strains to the intestinal mucosa and interaction with pathogens. Best Pract Res Clin Gastroenterol 2003, 17(5):741-754.

24. Banerjee P, Merkel GJ, Bhunia AK: Lactobacillus delbrueckii ssp. bulgaricus B-30892 can inhibit cytotoxic effects and adhesion of pathogenic Clostridium difficile to Caco-2 cells. Gut Pathog 2009, 1(1):8.

25. Servin AL: Antagonistic activities of lactobacilli and bifidobacteria against microbial pathogens. FEMS Microbiol Rev 2004, 28(4):405-440.

26. Soderling EM, Marttinen AM, Haukioja AL: Probiotic lactobacilli interfere with Streptococcus mutans biofilm formation in vitro. Curr Microbiol 2011, 62(2):618-622

27. Harith JF, Al- M, Saher Daher Abed H: Inhibitory Effect of Lactobacilli isolate on klebsiella pneumoniae Biofilm. Iraqi Academic Scientific Journals 2012, 11(2):168-179.

28. Maldonado NC, Silva De R, et al: A simple technique to detect klebsiella biofilm forming strains. Inhibitory potential of Lactobacillus Fermentum CRL 1058 whole cells and products. In Communicating current research and educational topics and Trends in Applied Microbiology. 2007:52-59.

29. Weinstein DL, ONeill BL, Hone DM, Metcalf ES: Differential early interactions between Salmonella enterica serovar Typhi and two other pathogenic Salmonella serovars with intestinal epithelial cells. Infect Immun 1998, 66(5):2310-2318.

30. Thirabunyanon M, Thongwittaya N: Protection activity of a novel probiotic strain of Bacillus subtilis against Salmonella Enteritidis infection. Res Vet Sci 2012, 93(1):74-81.

31. Wallis TS, Galyov EE: Molecular basis of Salmonella-induced enteritis. Mol Microbiol 2000, 36(5):997-1005.

32. Boyen F, Pasmans F, Van Immerseel F, Morgan E, Adriaensen C, Hernalsteens JP, Decostere A, Ducatelle R, Haesebrouck F: Salmonella Typhimurium SPI1 genes promote intestinal but not tonsillar colonization in pigs. Microbes Infect 2006, 8(14-15):2899-2907.

33. De Keersmaecker SC, Marchal K, Verhoeven TL, Engelen K, Vanderleyden J, Detweiler CS: Microarray analysis and motif detection reveal new targets of the Salmonella enterica serovar Typhimurium HilA regulatory protein, including hilA itself. J Bacteriol 2005, 187(13):4381-4391.

34. Datsenko KA, Wanner BL: One-step inactivation of chromosomal genes in Escherichia coli K-12 using PCR products. Proc Natl Acad Sci U S A 2000, 97(12):6640-6645.

35. Altier C: Genetic and environmental control of Salmonella invasion. J Microbiol 2005, 43:85-92.

36. Vishwakarma V, Periaswamy B, Bhusan Pati N, Slack E, Hardt WD, Suar M: A novel phage element of Salmonella enterica serovar Enteritidis P125109 contributes to accelerated type III secretion system 2-dependent early inflammation kinetics in a mouse colitis model. Infect Immun 2012. 80(9):3236-46.

37. Marathe SA, Ray S, Chakravortty D: Curcumin Increases the pathogenicity of Salmonella enterica serovar Typhimurium in murine model. Plos One 2010, 5(7):e11511.

doi:10.1186/1757-4749-5-11

Cite this article as: Das et al.: In vitro evaluation of anti-infective activity of a Lactobacillus plantarum strain against Salmonella enterica serovar Enteritidis. Gut Pathogens 2013 5:11. 